\title{
Ground- and Space-Based Observation of Kordylewski Clouds
}

\author{
Peng Wang $\mathbb{D},{ }^{1,2}$ Xiao-Jun Jiang, ${ }^{3,4}$ Xi-Yun Hou, ${ }^{5}$ Li-Hua Zhang $\mathbb{D},{ }^{1}$ Li-Xiang Jiang, \\ Jia-Qi Wang $\mathbb{D}^{3,4}$ Hui Zhi ${ }^{3},{ }^{3,4}$ Zi-Long Jiao $\mathbb{D}^{6}{ }^{6}$ Tao Li, ${ }^{6}$ Ming-He Liu $\mathbb{D}^{1}{ }^{1}$ \\ and Jian-Feng Wang $\mathbb{D}^{3}$
}

${ }^{1}$ DFH Satellite Co., Ltd., Beijing 100094, China

${ }^{2}$ School of Aerospace Engineering, Beijing Institute of Technology, Beijing 100081, China

${ }^{3}$ National Astronomical Observatories, Chinese Academy of Sciences, Beijing 100101, China

${ }^{4}$ School of Astronomy and Space Science, University of Chinese Academy of Sciences, Beijing 100101, China

${ }^{5}$ Nanjing University, Nanjing 210023, China

${ }^{6}$ Beijing Institute of Spacecraft Environment Engineering, Beijing 100094, China

Correspondence should be addressed to Peng Wang; wpp_p@139.com

Received 18 November 2020; Accepted 5 January 2021; Published 27 February 2021

Copyright (C) 2021 Peng Wang et al. Exclusive Licensee Beijing Institute of Technology Press. Distributed under a Creative Commons Attribution License (CC BY 4.0).

\begin{abstract}
The ghost dust clouds in the vicinity of Earth-Moon triangular libration points are known as the "Kordylewski clouds" (KDCs). Objects in the KDCs may give hints to the physical, chemical, and dynamical properties of the solar system. As a result, in situ exploration of the KDCs can help us understand the evolution of our solar system. This paper first summarizes the observation history of the KDCs. Based on the properties of the KDCs, a ground- and space-based observation concept is proposed, using the CAST 100 platform developed by DFH and ground stations. Some details of the concept are exploited.
\end{abstract}

\section{Introduction}

Dust is one of the main components of the universe and plays a very important role in the formation and evolution of galaxies and stars [1]. Dust in the solar system is mainly composed of remnants of the planetary disk of the protosolar system, the debris produced during the dynamic evolution of the solar system, and a very small amount of interstellar dust. Due to the orbital cleaning effect of massive planets, nowadays, there is little protoplanetary disk material left near the planets. About $1 \%$ of the dust in the solar system is interstellar dust. Therefore, it is possible to obtain samples of interstellar dust by in situ detection of the cosmic dust.

Dust is widespread in the universe. Figure 1 shows a snapshot of the dynamic distribution of some main comet tail particles in the solar system [2]. In 1930, Trumpler used observations of starlight absorption and reddening in the direction of a galactic disc to clearly prove the existence of cosmic dust for the first time [3]. Triangular libration points of the Earth-Moon system may host abundant pure cosmic dust. Confirming its existence will open up a new way for humans to directly explore the interstellar and interplanetary medium.

In 1772, the French Mathematician Joseph Louis Lagrange pointed out the five special invariant relative configurations (also known as the central configuration) of the general three-body problem. The reduced case of the general three-body problem, where two massive bodies revolve with each other in circular orbits and the third body is assumed massless, is called the circular restricted three-body problem (CRTBP). The three collinear and the two equilateral central configurations of the general three-body problem are now reduced to three collinear libration points L1, L2, and L3 and the two triangular libration points L4 and L5 in the CRTBP, respectively. In the CRTBP model of the EarthMoon system, the two triangular libration points (hereafter, we refer them as EML4 and EML5) are stable, and dust particles may be gravitationally trapped around them to form the KDCs. An illustrative picture of the distribution of the KDCs is given in Figure 2. The KDCs can serve as a very useful experimental field for interplanetary dust particles (IDP) and interstellar dust (ISD). There are unique advantages of 


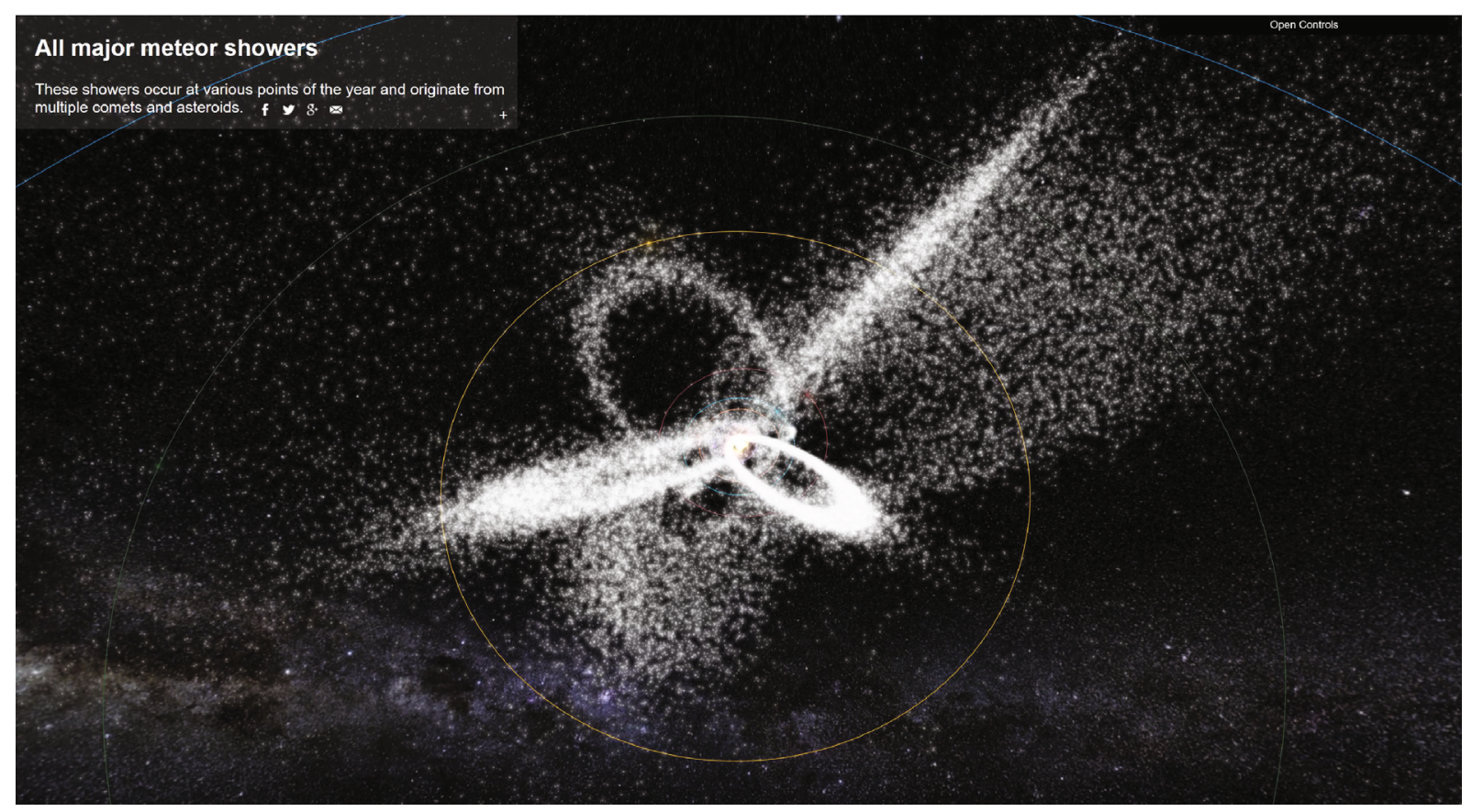

FIGURE 1: A snapshot of the dynamic distribution of some main comet tails in the solar system which is accessible via the link [2].

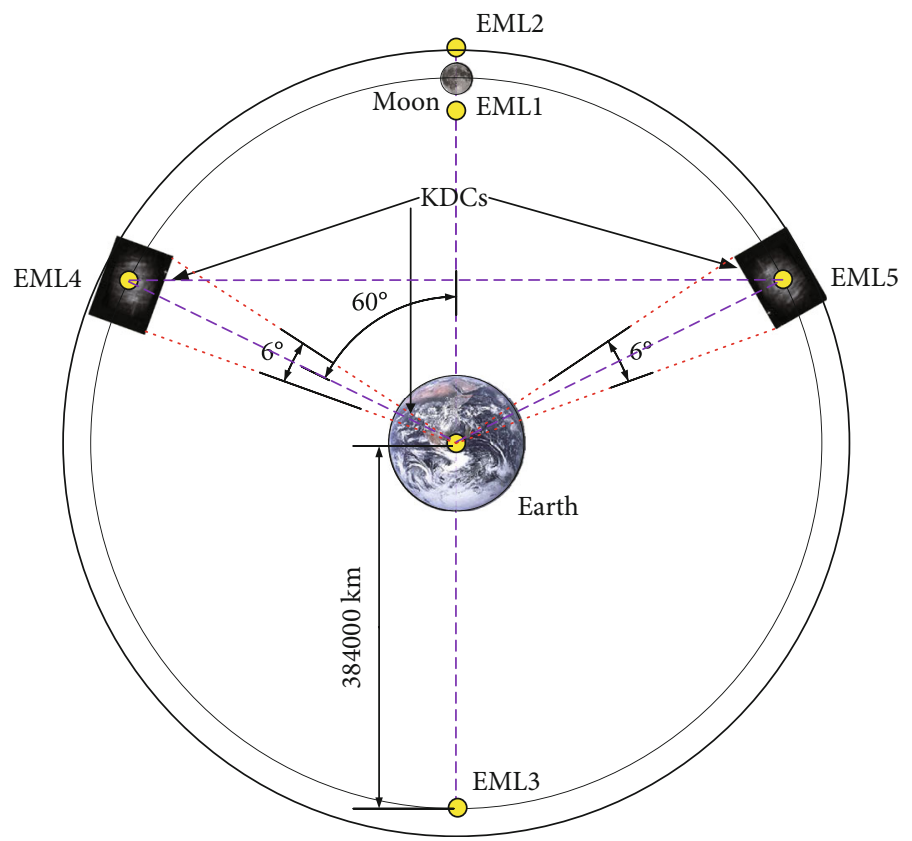

FIGURE 2: The distribution geometry of the KDCs in the Earth-Moon system.

the KDCs. (1) Their flux should be relatively higher than the average flux of dust particles in the Earth-Moon system, which is helpful for their detection; (2) there is an energy barrier between the vicinity of Earth and the space around the triangular libration points. As a result, the KDCs are prevented from the pollution from Earth's vicinity and should be cleaner than the dust samples in the vicinity of Earth. Here, "cleaner" means that the dust samples are not mixed with dust particles due to humanity's space activities around the Earth. (3) The mean distance between Earth and the
KDCs is about $380000 \mathrm{~km}$, which is not very far. Even using the low-energy transfer, it can be reached in $\sim 100$ days, so the launch and time cost of missions to the KDCs are lower than those to the interplanetary space. Besides the above advantages, with more and more missions being planned to visit these points in the future, the space environment of the Earth-Moon system for which the KDCs is just one of the topics is an urgent need for future utilization of these points.

Ground and space observations, along with some theoretical analysis indicate that the KDCs are currently a 
dynamical balance of dust particles. Their structure may change with both the space and the time. Such a timeand space-varying structure may explain the fact that observations of the KDCs can be hardly repeated by ground stations. This also challenges the space-based observations. How to choose the right time and the right position to encounter the KDCs is a key problem to the space-based detection missions.

\section{Current Observation Status of the KDCs}

In 1906, as a direct validation of the stability of the triangular libration points, the first Trojan asteroid Achilles was found being trapped around the triangular libration points of the Sun-Jupiter system. The Discovery of more and more Trojan asteroids attracted people's attention on the research and observation of the KDCs in the Earth-Moon system. Researchers attack this problem from both theoretical analysis of the dynamics and observations.

Theory on the dynamics of the KDCs mainly focuses on the origin and distribution of the KDCs. However, little literature is found on their origin. One possible origin is that the KDCs are caused by dust particles from the interplanetary space. For Trojan asteroids in the Sun-Jupiter system, some researchers used the well-known NICE model to explain the dynamical capture of the asteroids in the early history of the solar system [4]. This theory is later modified by the improved NICE model [5]. Also, the well-known PoyntingRobertson (P-R) effect gradually drags dust particles inwards to the Sun [6]. Although the KDCs are in the Earth-Moon system, which is not a Sun-planet system like the SunJupiter system, these previous studies may help us build capture mechanisms of the KDCs. One problem which hopefully can be answered by the capture theory of the KDCs is the constant source of the KDCs.

Since 1875, represented by Routh, Lyapunov, Arnold, Leontovich, and Deprit, mathematicians studied the stability of the libration points. Routh found that triangular libration points of the Earth-Moon system were stable in the linear sense [7]. The model composed of the Sun, Earth, and Moon, i.e., the Sun-Earth-Moon model, is currently the main model to study the stability of the triangular libration points of the Earth-Moon system. Some special solutions based on this model are given by Kamel [6], Simó et al. [8], and Hou and Liu [9]. Using the numerical method to survey spatial orbits around the triangular libration points, Jorba pointed out some inclined orbits with respect to the Moon's orbital plane that may stay around the triangular libration points for hundreds of years [10].

In 2018, Balogh simulated the dynamical distribution of the KDCs in the spatial four-body model composed of the Sun, the Earth, the Moon, and the dust particle. Their simulated distribution satisfactorily agrees with their observations, which gives a firm support to the opinion that the KDCs are a time- and space-varying population. In 2019, Salnikova and Stepanowa stated that the simulated results of their theoretical model agree with the observations. Due to this finding, they are strongly in favor of the opinion that KDCs are time- and space-varying [7]. All these theoretical studies indicate that dynamics around the Earth-Moon triangular libration points are extremely complicated. We cannot exclude the possibility that dust particles can temporarily or long-termly stay around them. Here, when we mention "temporarily," we mean the dust particles can stay around the triangular libration points for about one Moon's orbital period ( month). When we mention "long-termly," we mean that the dust particles can stay around the triangular libration points for about one year or even longer. Some examples are shown in Figure 3. The dust particles are assumed chargeless and initially static at the triangular libration points. Depending on their sizes, they receive solar radiation forces (solar radiation pressure, solar wind, and P-R drag) of different strength, and their lifetime around the triangular libration point varies significantly, from less than one month to tens of years. Figure 3 only serves as an example to show the complex dynamics of the dust particles around the triangular libration points. In reality, the case is even more complex because dust particles are usually charged and their charges may change with time. Moreover, the time-varying electromagnetic field may influence the trajectory of a single dust particle, making it different from the one that is influenced only by gravity, and influence the overall distribution and stability of the KDCs. Currently, we are still working on the dynamics of charged dust particles.

One remark is made here. Current theoretical analysis does not focus much on the solar radiation pressure and the P-R effect, but researchers' (including the authors of this manuscript) numerical simulations indicate that dust particles can still stay around the Earth-Moon triangular libration points when these effects are included, i.e., dust particles move on orbits around the triangular libration points. These results also provide the basis for orbit design of the in situ detection missions.

At present, ground-based observation is the main method to observe the KDCs. Since 1951, Kordylewski began to search for objects around triangular libration points of the Earth-Moon system. Until 1961, between 6 March and 6 April, Kordylewski succeeded in photographing two bright patches around the Earth-Moon L5 point [7]. Besides Kordylewski's observation, there are also some other successful observations. Simpson claimed successful observations of the KDCs by using the Kuiper Airborne Observatory in 1967 [11]. With the help of the Orbiting Solar 40 Observatory, Roach also detected the KDCs in 1975 [12]. In 1989, the clouds were again photographed by Winiarski [13]. On the other hand, many scholars are strongly skeptical about the existence of the KDCs (Roosen, Harrington \& Jeffreys 1967; Bruman 1969; Valdes \& Freitas 1983; Igenbergs et al. 2012) [14]. According to these scholars, the KDCs cannot exist around the EML4 and EML5 points for long due to the gravitational perturbation from the Sun, solar wind, and other planets [14].

Although the KDCs cannot exist for a long time, they still have the chance of short-term survival. From June to October in 2017, Slíz-Balogh et al. [14] made successful observations by using ground-based polarized light photography technology. Through a series of innovative methods, the important indirect evidence of dynamic stability of the KDCs is 


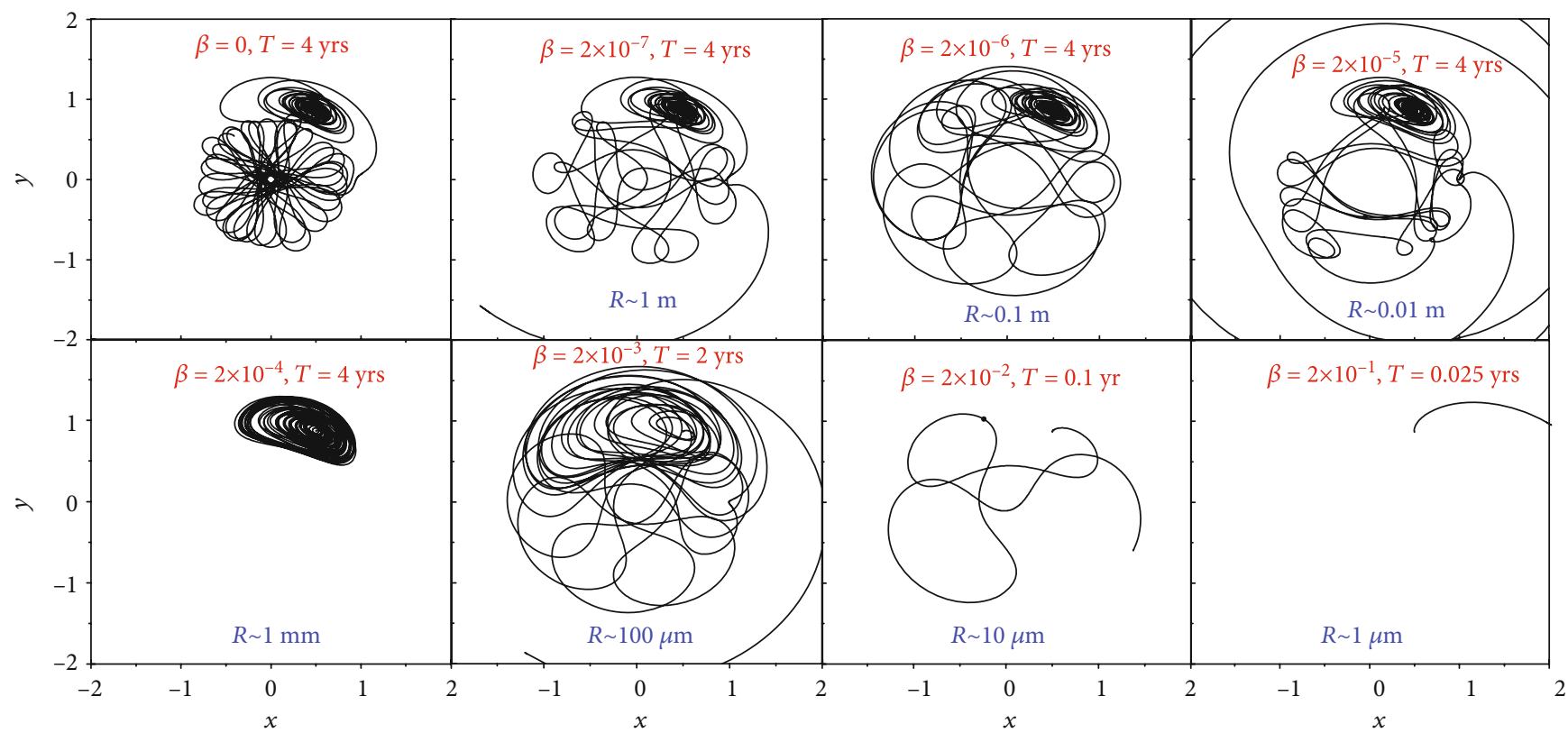

FIgURE 3: Stability analysis of the KDC dust particles with different sizes. The dust particle is assumed chargeless and initially static at the triangular libration point L4. With the joint influence from the gravity of Earth, Moon, and Sun and the solar radiation forces (solar radiation pressure, solar wind, $\mathrm{P}-\mathrm{R}$ drag), the trajectory shows great differences for particles with different sizes. In the figure, $\beta$ is a parameter of the solar radiation forces which is inversely proportional to the particle's size. Unit in these frames is the instantaneous Earth-Moon distance.

TABLE 1: KDC ground-based observation.

\begin{tabular}{|c|c|c|c|c|c|}
\hline Observer & Date & Technique & $\begin{array}{l}\text { Magnitude } \\
\left(\operatorname{arcsec}^{-2}\right)\end{array}$ & $\begin{array}{l}\text { Cloud } \\
\text { size }\end{array}$ & $\begin{array}{l}\text { Density } \\
\left(\mathrm{cm}^{-2}\right)\end{array}$ \\
\hline Grün et al. 1985 & & - & - & $5^{\circ}$ & $\sim 10^{-3}$ \\
\hline \multirow[t]{2}{*}{ Kodylewski 1961} & Mar. Apr. 1961 & $\begin{array}{c}\text { Photographic ( } 35 \mathrm{~mm} \text { film, } \\
50 \mathrm{~mm} \text { focal length, f/1.5, } 12 \text { min.exp.) }\end{array}$ & 22 & $5^{\circ}$ & $2.0 \times 10^{2}$ \\
\hline & Jan. 1964 & Visual & & & \\
\hline \multirow[t]{2}{*}{ Simpson $1967[11]$} & Feb. 1966 & Photographic (4 8 min exp.) & 20.5 & $4^{\circ}$ & $6.8 \times 10^{2}$ \\
\hline & Mar. 1966 & Visual (airborne) & & & \\
\hline Vanysek 1969 & Mar. 1966 & Visual (airborne) & 22.6 & $4^{\circ}$ & $1.0 \times 10^{2}$ \\
\hline Roosen 1968 & Mar. 1966, Mar. 1967 & $\begin{array}{c}\text { Photographic (35 } \mathrm{mm} \text { film, } \\
50 \mathrm{~mm} \text { focal length, } \mathrm{f} / 1.4,1 \sim 9 \mathrm{~min} . \exp \text { ) }\end{array}$ & $\begin{array}{l}\text { Fainter } \\
\text { than } 26\end{array}$ & $<2^{\circ}$ & $<5.4$ \\
\hline Wolff et al. 1967 & Mar. 1967 & $\begin{array}{l}\text { Photographic (airborne, } 35 \mathrm{~mm} \text { film, } \\
50 \mathrm{~mm} \text { focal length, f/1.4, 20 40 min.exp) }\end{array}$ & $\begin{array}{l}\text { Fainter } \\
\text { than } 25.4\end{array}$ & $<2^{\circ}$ & $<9.2$ \\
\hline Roach 1975 [12] & $\begin{array}{l}\text { Autumn } 1969 \\
\text { to Winter } 1970\end{array}$ & $\begin{array}{c}\text { Photometrric: Rutgers Zodiacal Light } \\
\text { Analyzer on OSO-6 }\end{array}$ & 24.5 & $6^{\circ}$ & 21 \\
\hline Schlosser et al. 1975 & Mar. to Apr. 1971 & Photographic: using wide angle $\left(140^{\circ}\right)$ photographs & $\begin{array}{l}\text { Fainter } \\
\text { than } 26.5\end{array}$ & $6^{\circ}$ & $<3.1$ \\
\hline Munro et al. 1975 & Jul. to Dec. 1973 & Photometric (white light coronagraph on Skylab) & $\begin{array}{l}\text { Fainter } \\
\text { than } 16.7\end{array}$ & $<7^{\circ}$ & $<1$ \\
\hline Winiarski 1989 [13] & Feb. 1976 & Photographic: various techniques & 23.8 & - & 30 \\
\hline Amanda 2010 & Jun. 2010 & CCD & - & - & - \\
\hline Slíz-Balogh 2017 & Jun. to Oct. 2017 & Polarization CCD, private observatory & - & $15^{\circ} \times 10^{\circ}$ & - \\
\hline
\end{tabular}

obtained [14]. Table 1 shows the ground observation results in recent 70 years [15].

According to ground-based optical observations, the spatial distribution and density of the KDCs can be preliminarily estimated. In case that the KDCs do exist around the EarthMoon triangular libration points, the density is estimated to be about $100 / \mathrm{cm}^{2}$, with a spatial distribution within $6^{\circ}$ when viewing from the Earth. In case that there is no obvious 


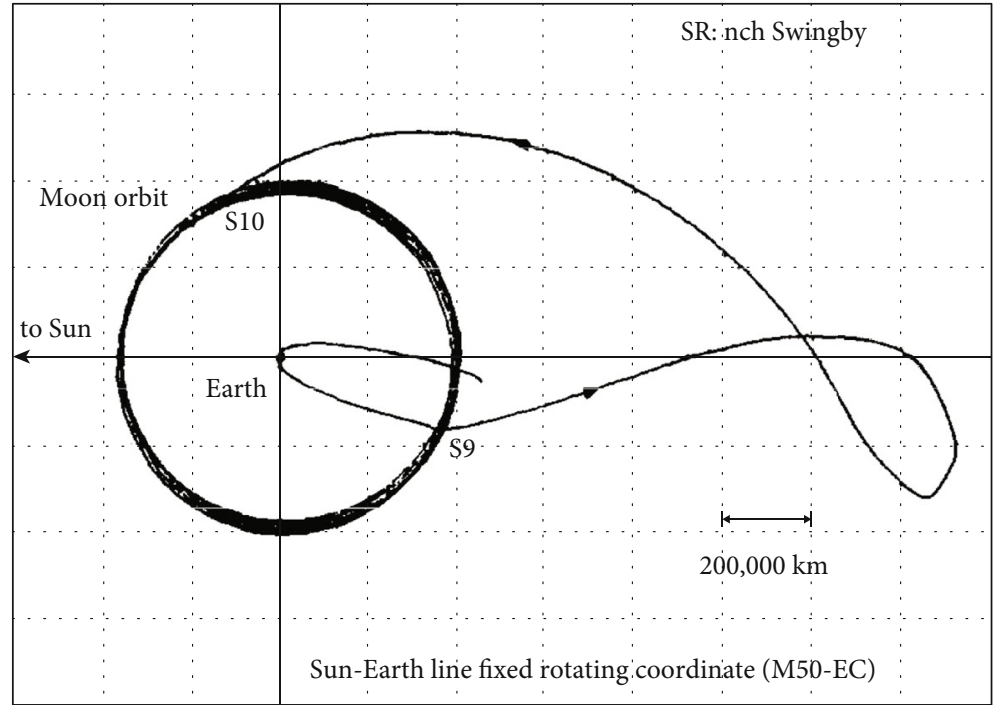

FIGURE 4: Trajectory of the HITEN spacecraft between its $9^{\text {th }}$ and $10^{\text {th }}$ lunar swingby. The circle indicates Moon's orbit. S9 and S10 indicate the place where the swingby happens (from Ref. [18], with copyright permission).

accumulation of dust particles around the Earth-Moon triangular libration points, the density is estimated to be about $5 \sim 30 / \mathrm{cm}^{2}$. The difference in the brightness between the background and the KDCs is less than 1 magnitude.

Compared with ground-based observations, in situ space-based observations can provide more detailed information about the KDCs and produce more scientific results.

In 1963, Kliore in JPL for the first time proposed in situ exploration proposal of the KDCs [16]. Two types of proposals were proposed. One was flyby and the other was rendezvous. In 1967, Wheeler proposed to use the backup of the Apollo spacecraft and newly developed optical payloads for in situ detection of the KDCs [17]. Wheeler thought the KDCs were mainly composed of very primitive materials, some of which were hardly accessible to us.

In 1988, Ryden proposed a flyby exploration proposal of the KDCs, based on ion electric propulsions. The total weight of the probe was $300 \mathrm{~km}$, with $240 \mathrm{~km}$ of the dry mass and $60 \mathrm{~kg}$ of the propellant. The probe was planned to be initially launched to an orbit with an apogee height of about the lunar orbit height. The low-thrust propulsion gradually leveraged the perigee height to the lunar orbit and finally sent the probe to a rendezvous orbit around one of the Earth-Moon triangular libration points.

In 1991, Japan started their Moon exploration program [18]. The spacecraft HITEN weighs about $197 \mathrm{~kg}$ in total (42 kg for the propellant). On $2^{\text {nd }}$ Oct. 1991, the probe made a first attempt of the in situ detection of the KDCs. The spacecraft's trajectory in the Sun-Earth and the EarthMoon synodic frames is shown in Figures 4 and 5. After the $10^{\text {th }}$ lunar swingby (see Figure 4), the spacecraft began its second phase (see Figure 5) and visited the EML4 point first and then the EML5 point. The rendezvous time with the EML4 and the EML5 is approximately three months, with only one loop around each libration point. During the rendezvous, the dust counter onboard the probe did not find any obvious accumulation of dust particles around these points. Nevertheless, this finding does not completely rule out the existence of the KDCs, since the probe revolves around each libration point for only one loop.

In 1992, Lorenz et al. proposed a flyby proposal based on chemical propulsions to detect the KDCs. The total weight of the probe was $305 \mathrm{~kg}$, with $182 \mathrm{~kg}$ for the dry mass and $123 \mathrm{~kg}$ for the propellant. The probe would firstly fly to a GTO orbit and then make multiple flybys of the EML4 and EML5 points.

In 1993, an extension of the Giotto mission was proposed to detect the KDCs [15]. The Giotto probe was originally designed to visit the comet Halley. The probe weight is $960 \mathrm{~kg}$ in total $(570 \mathrm{~kg}$ for the dry mass and $390 \mathrm{~kg}$ for the propellant). Only the piezoelectric momentum sensors could be used to detect the KDCs, and the ability of this payload was limited.

In 2007, Universitaet Stuttgart, Germany, proposed to detect the KDCs during the Earth-Moon transfer phase in their lunar swingby conceptual study [19]. The spacecraft weighed $200 \mathrm{~kg}$ in total, and the payload was about $30 \sim 40 \mathrm{~kg}$.

In 2012, Lightsey proposed to detect the KDCs using a cube satellite flying formation [20]. A set of cube satellites was packed together and sent to the Earth-Moon transfer orbit. The low-thrust Xenon thruster gradually increased the height of the perigee, and the cube satellites were deployed to make flyby observations of the KDCs.

In the past 70 years, ground-based optical observation is the main means to detect the KDCs. Due to the long distance from the KDCs, the ground-based observation can detect a wide region around the triangular libration points. As a result, it is suitable to study the varying properties of the KDCs in large scales. Due to influences from the weather, galactic light, starlight, ecliptic light, atmospheric glow, and the inherent time- and space-varying property of the KDCs, it is difficult to effectively repeat the observations for ground-based observations. The ground-based polarization technique has made 


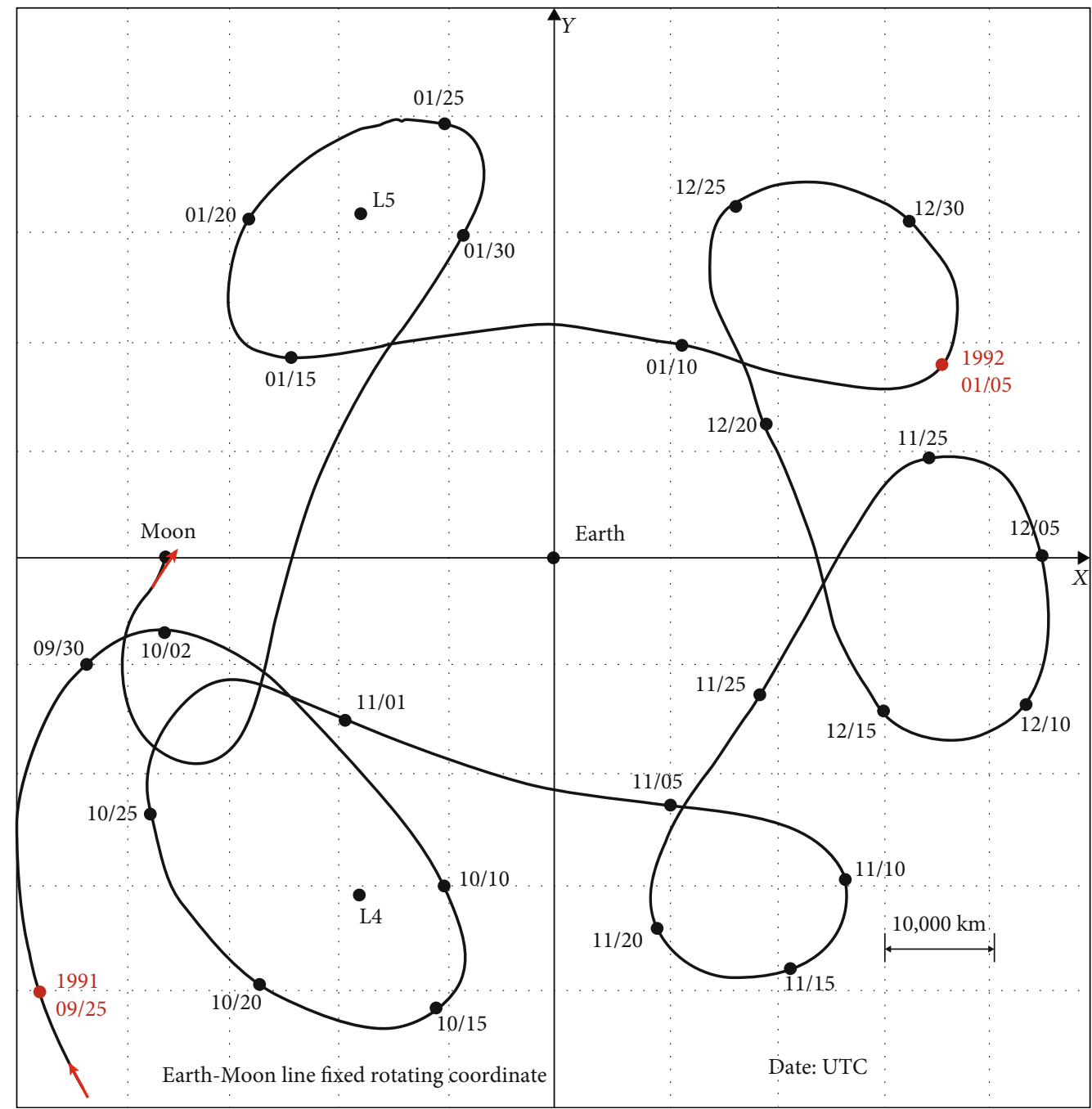

FIGURE 5: L-point excursion trajectory of the HITEN spacecraft. The coordinate is the Earth-Moon rotating frame, and the spacecraft moves counterclockwise in this frame (reproduced from Ref. [18], with copyright permission).

some positive progress in the observation of the KDCs, but still, it needs further verification.

Compared with ground-based observations, space-based in situ detection can obtain more details on the properties of the KDCs.

Generally, there are two types of space-based observations. One type is rendezvous. The spacecraft rendezvouses around the libration point and makes long-term in situ observations of the KDCs. The other type is flyby. The spacecraft makes several passages through the region around the libration points with a high relative speed in short-time scales to make repeated in situ observations of the KDCs. The main disadvantage of space-based observation is that only a small portion of the space can be visited by the probe at a specific time, which is unfavorable for the KDC detection due to their large-scale space- and time-varying properties. The rendezvous approach can help increase the possibility of detection, but the relative speed between the spacecraft and the dust particles usually does not exceed $600 \mathrm{~m} / \mathrm{s}$, so conventional dust counters based on high-speed impact are no longer valid. The relative low speed between dust particles tempo- rarily trapped in the Earth-Moon system and HITEN is one of the possible reasons for its failure to detect them. That is the reason why we need a dust detection instrument that can detect low-speed dust particles.

\section{The Joint Ground- and Space-Based Observation Plan}

When viewed from the Earth, the KDCs distribute in a region of $\pm 3^{\circ}$ wide around the triangular libration points. To study the space- and time-vary laws of the KDCs, an observation plan using both the ground-based and space-based facilities is proposed. The scientific objectives of the mission include the following:

(1) Explore the dynamic spatial distribution of the KDCs, their orbital stability, and density

(2) Measure the electrical charges, mass, flux density, and environmental parameters of the dust particles 
(3) Analyze the size and components of the dust particles that compose the KDCs according to the observations. This will provide clues for researchers to understand the capture and evolution mechanism of the KDCs

The engineering objectives of this mission include the following:

(1) Confirm the environment parameters is the cis-lunar space (including the triangular libration points)

(2) Validate the rendezvous technology around the Earth-Moon triangular libration points

(3) Test the in situ dust detection technology that covers a wide range of dust speeds

3.1. The Space-Based Observation. For the space-based observations, based on the results of ground-based observations, some regions where the KDCs may exist are firstly selected. The spacecraft's orbit is then adjusted to fly through these regions to confirm the existence of the KDCs, by comparing the dust particle fluxes of these regions and nearby regions. At the final stage of the mission, the spacecraft rendezvouses with the Earth-Moon libration point.

The whole mission is split into four phases as follows:

The Earth-Moon transfer phase. The spacecraft is firstly sent to an Earth-Moon transfer orbit. Then, the spacecraft adjusts its orbit to enter a highly elliptic orbit around the Earth.

The flyby observation phase. Using the difference in the orbital period of the spacecraft and the Moon, the spacecraft flies by the EML4 and EML5 points. With a small amount of control, the spacecraft can adjust its orbit through prescribed regions around the EML4 and EML5 points where the KDCs may reside.

Libration point transfer phase. After several flybys, the spacecraft maneuvers to approach the Moon, makes a lunar swingby, and flies to the EML4 point.

Rendezvous phase. With a small maneuver, the spacecraft is inserted into a "patrol" orbit around the EML4 point that covers a large region around the triangular libration point without orbit control [21]. The spacecraft moves on this orbit for at least 3 years, exploiting the distribution, dynamics, physics composition, etc. of the KDCs.

Once the spacecraft is launched, it starts its observation of dust particles en route to the libration points. The flyby observation phase and the rendezvous phase in the EarthMoon's synodic frame are shown in Figures 6 and 7.

The total $\Delta v$ cost is estimated to be around $580 \mathrm{~m} / \mathrm{s}$. The spacecraft is designed using the platform CAST 100 developed by DFH. The ADN propulsion is used, with a specific impulse of $230 \mathrm{~s}$. A maximum of $1000 \mathrm{~m} / \mathrm{s}$ is planned. The payloads weigh $10 \mathrm{~kg}$, which can fulfill the goal of the mission.

The dust detection payload is designed to be able to detect dust particles with both low and high speeds. The payload utilizes a composite detection method which includes electrostatic grids and metallic target. The grids were used to measure parameters of particles with low speed, and the

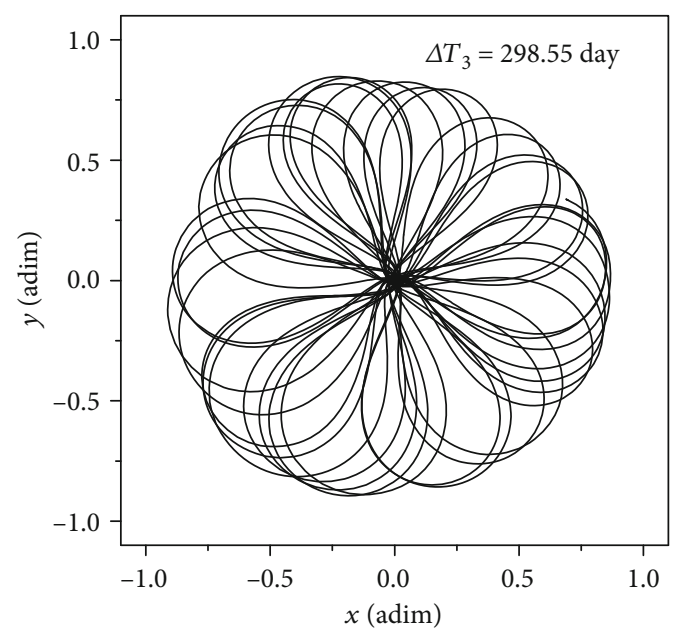

Figure 6: Trajectory of the flyby phase. Unit in the figure is the instantaneous Earth-Moon distance.

target-for particles with high speed. Some main parameters of the dust detection payload are listed in Table 2.

3.2. The Ground-Based Detection. The dust phenomena that can be observed from the Earth mainly include the zodiacal light and the counterglow (similar brightness as the KDCs [22]). In addition to the influence of the Earth's atmosphere, these are also the main interference factors for observing KDCs. Due to the aggregation of dust particles at EML4 and EML5, it is currently thought that KDCs at these locations are denser. The relatively short-lived, cooler dust clouds have significant effects of extinction, scattering, and polarization. Ground-based observation should be conducted from the following three perspectives:

(1) Extinction: the dust clouds have an absorption effect on the surrounding radiation field. By comparing the reddening effects of two stars with the same spectral type due to dust clouds, the extinction curves of dust clouds can be measured, and particle size distribution and shape can be simulated and analyzed according to the extinction intensity of different wavelengths. According to the theory, the KDCs are mainly composed of the dust from the solar system protoplanetary disk. By observing the spectra of stars of the same spectral type before and after being "eclipsed" by the KDCs, analyzing the extinction curves of KDCs, and comparing the difference between the extinction curve of the KDCs and the extinction curve of interstellar dust (as shown in Figure 8), a reference to the composition of the KDCs can be formed and the origin of KDCs can be further inferred [3]

(2) Scattered radiation: according to the extinction curve, the dust influence on near-infrared and visible light is mainly scattering. It is possible to obtain optical characteristics of dust particles and the information of the particle size and chemical composition by studying the scattering characteristics of the dust. Previous 


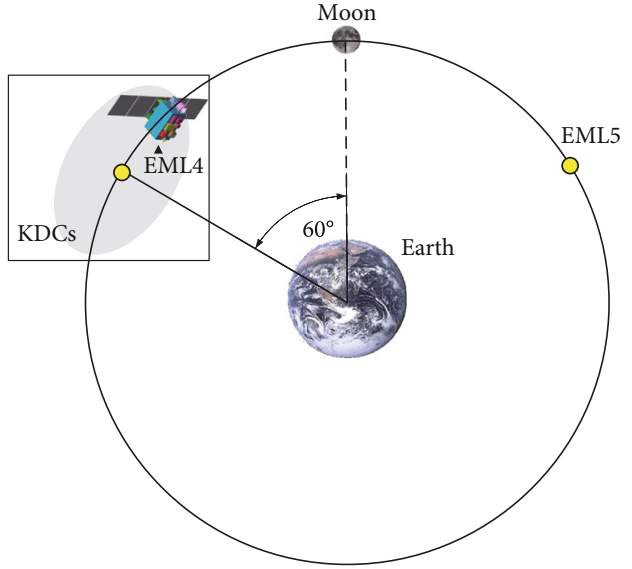

(a)

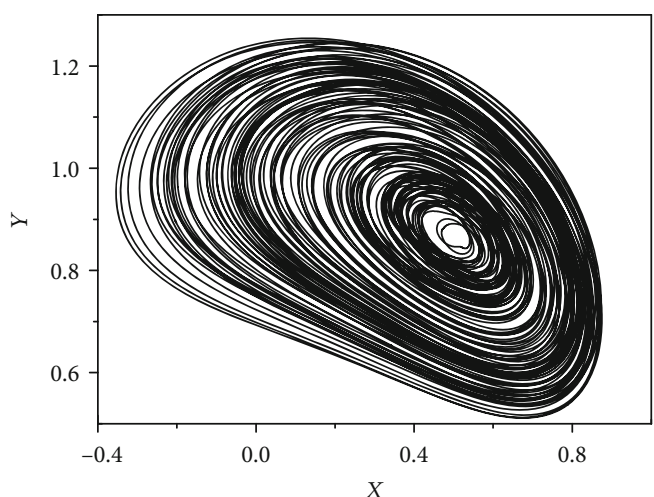

(b)

FIGURE 7: Trajectory of the rendezvous phase. The EML4 point is taken as an example. The region the spacecraft can cover in the rendezvous phase is illustrated by the gray region in the left frame of the figure. The right frame shows the rendezvous trajectory around the EML4 point. Unit in the right figure is the instantaneous Earth-Moon distance.

TABLE 2: Parameters of the spacecraft and the payload.

\begin{tabular}{lc}
\hline Platform & $\begin{array}{c}\text { Parameters } \\
\text { Launch orbit }\end{array}$ \\
Mission orbit & $\begin{array}{c}\text { Lunar transfer orbit (LTO) } \\
\text { Highly elliptic orbit and } \\
\text { rendezvous orbit around EML4 }\end{array}$ \\
Total mass & $100 \mathrm{~kg}$ \\
Propulsion system & The ADN nontoxic propulsion \\
Total $\Delta v$ budget & $1000 \mathrm{~m} / \mathrm{s}$ \\
Power requirement & $200 \mathrm{~W}$ \\
Attitude control accuracy & Better than $0.01^{\circ}$ \\
Life & 4 years \\
\hline Payload & Parameters \\
Range of measurable velocity & $1-30000 \mathrm{~m} / \mathrm{s}$ \\
Velocity direction & Better than $5^{\circ}$ \\
measurement accuracy & $5 \times 10^{-12}-5 \times 10^{-6} \mathrm{~g}$ \\
Dust particle mass & $1 \times 10^{-16}-1 \times 10^{-13} \mathrm{C}$ \\
Electric charge & $0.75 \mathrm{~m}^{2}$ \\
Detection area & $10 \mathrm{~kg}$ \\
Mass &
\end{tabular}

observations showed that the brightness of the KDCs is fainter than 22 magnitudes [24]. Therefore, direct observation of the scattered radiation of the KDCs requires very strict requirements on the skylight of the observation site, and the measurement accuracy better than 0.01 magnitude, it is crucial to improve the signal-to-noise ratio as much as possible within the limited integration time. According to the current theories, KDCs' matter will maintain dynamic balance continuously during the Moon's revolution, and its morphology may constantly change at the same time. By comparing images with a certain integration time (ensuring that the Analog To Digital Unit (ADU) readings of the images are in the linear

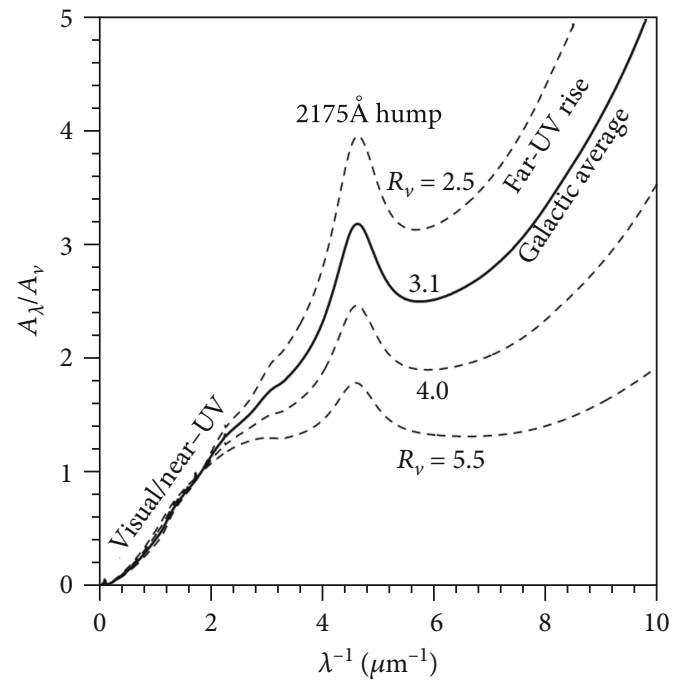

FIGURE 8: Interstellar extinction curve. The ordinate is the extinction coefficient, and the abscissa is the wavelength (adapted from Ref. [23]).

region), these morphology changes can be analyzed. During the observations, it is necessary to set the parameters of the detector with a higher dynamic range to improve the observation accuracy

(3) Polarization: according to the Mie scattering theory, the sunlight scattered by dust is partially linearly polarized light. The degree of polarization (DoP) is related to the scattering angle, particle shape, size, density, and material of the dust. By observing the polarization characteristics of the KDCs, we can understand their distribution and physical characteristics. Extending current studies of atmosphere polarization characteristics to the KDCs, the scattering angle is the solar phase angle when KDCs are observed. As shown in Figure 9, the solar phase angle 


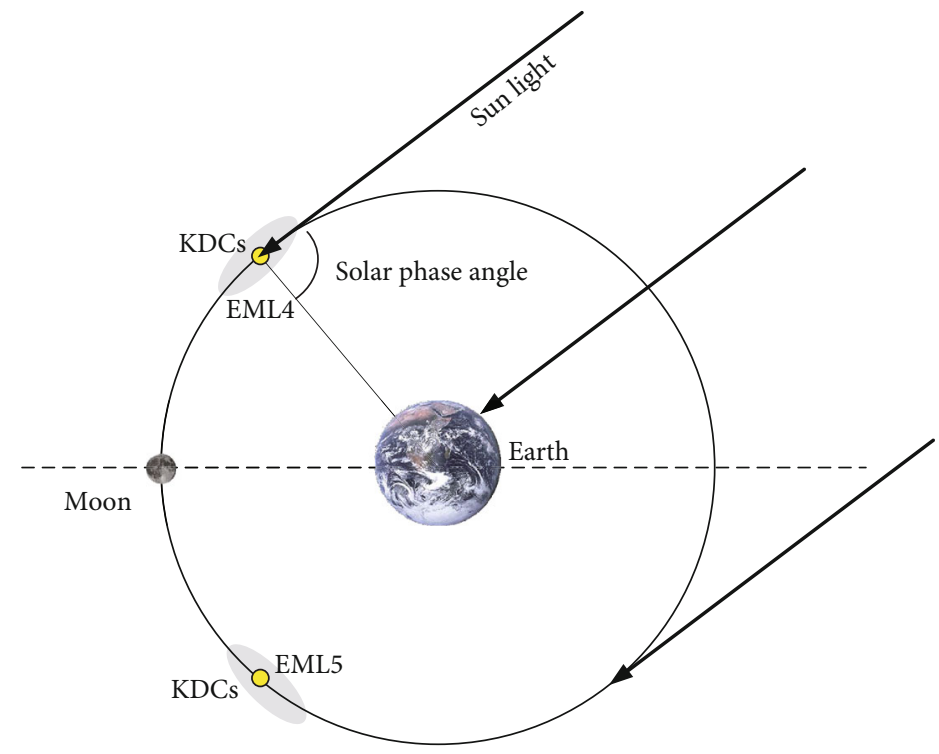

Figure 9: The solar phase angle during KDCs' observation.

is the angle formed by the lines connecting the observer and the Sun with KDCs as the apex. Since the KDCs revolve synchronously with the Moon, this angle will change with the lunar phase cycle. Therefore, the degree of polarization and the polarization angle (PlA) of the KDCs also change with the solar phase angle (as shown in Figure 9). The closer the scattering angle is to $90^{\circ}$, the higher the linear polarization degree is [25].

The atmospheric scattering effect of the night sky is small, so the polarization of the night sky light is much weaker than the daylight. Observing the KDCs when the DoP is relatively high may make the KDCs distinguished in the sky background. However, at that time, KDCs' visual position is close to the horizon and faces the interference of moonlight, so they are difficult to be observed. In addition, there are many artificial or natural factors that interfere with the KDC observations. We can choose observation sites or use filters to avoid artificial polarized light pollution, while atmospheric interference can be partially eliminated through the atmospheric polarization model reduction. Zodiacal light and counterglow have a completely different distribution from the KDCs; their DoP and PlA are approximate constants (because the solar phase angle of the zodiacal light and the counterglow is almost fixed), which keep stable with the observations at different phases of the moon, while the DoP and PlA of the KDCs will change with the lunar phase. Observations of polarization characteristics have become an important method to distinguish KDCs from various interference factors.

A typical astronomical polarization photometer uses Wollaston prism as a polarizer, and two detectors to simultaneously image the light of two polarization angle orientations, $0^{\circ} / 180^{\circ}$ and $45^{\circ} / 135^{\circ}$. The subsequent data reduction focuses on extracting the intensity of two channels, solving the Muller matrix to obtain the Stokes parameters, and obtaining the DoP and PlA ultimately.
3.3. The Joint Ground-and Space-Based Observation Plan. The present studies show that the KDCs are relatively thin dust clouds, composed of cooler dust particles. The accuracy of ground-based observations is very sensitive to the atmosphere and observation environment; the data of photometric observation usually has a large error. Photometry observation can only detect the change of KDCs' contour and shape; polarization observation can obtain the parameters of dust particle shape, size, etc., but need the aperture of a telescope larger than 2 meters.

Space-based observations can greatly compensate the limitation of ground-based observations. During the flyby phase of the mission, ground-based observations can be arranged to find the regions in space where the KDCs may reside, and the spacecraft can be guided to visit these regions. As a result, except the difference of the dust flux in different regions visited by the spacecraft can be obtained, the difference between ground-based and space-based observations of the same region can be obtained. Meanwhile, according to the data such as dust flux and charges obtained by spacebased observations, simulations and observations on the ground can be calibrated.

\section{Conclusions}

An observation plan of the KDCs is firstly proposed in this paper. The mission is composed of two parts. The first part is the ground-based observation. From the ground-based observation, we are able to characterize some global properties of the KDCs, such as their position in space. This information will guide the second part of the plan which is the space-based observation. The spacecraft will first flyby and then rendezvous with the Earth-Moon triangular libration points for the first time, making constant observations of the KDCs. In situ exploration of the KDCs is very important for both the dust science and libration point missions. Our plan can serve as a pioneer of the exploration and utilization program of the Earth-Moon triangular libration points. 


\section{Conflicts of Interest}

The authors declare that there are no conflicts of interest regarding the publication of this article.

\section{Acknowledgments}

This study is supported by the National Natural Science Foundation of China (11773004).

\section{References}

[1] D. E. Osterbrock, Astrophysics of gaseous nebulae and active galactic nuclei, Published by University Science Books, 1989, ISBN 0935702229.

[2] “All major meteor showers," https://www.meteorshowers.org/ view/all.

[3] M.-P. Li, Z. Gang, and A.-g. Li, "Interstellar dust: current status and perspectives," Progress in Astronomy, vol. 24, no. 3, pp. 260-276, 2006.

[4] H. P. Robertson, "Dynamical effects of radiation in the solar system," Monthly Notices of the Royal Astronomical Society, vol. 97, pp. 423-438, 1937.

[5] D. Nesvorny, D. VOkrouhlicky, and A. Morbidelli, "Capture of Trojans by jumping Jupiter," The Astrophysical Journal, vol. 768, no. 1, pp. 1-8, 2013.

[6] A. A. Kamel, Perturbation theory based on Lie transforms and its application to the stability of motion near Sun-perturbed Earth-Moon triangular libration points, Ph.D. thesis, Stanford University, 1969.

[7] T. Salnikova and S. Stepanov, "Existence of elusive Kordylewsky cosmic dust clouds," Acta Astronautica, vol. 163, pp. 138-141, 2019.

[8] C. Simó, G. Gómez, À. Jorba, and J. Masdemont, "The bicircular model near the triangular libration points of the RTBP," in From Newton to Chaos, A. E. Roy and B. A. Steves, Eds., pp. 343-370, Plenum Press, New York, 1995.

[9] X. Hou and L. Liu, "On quasi-periodic motions around the triangular libration points of the real Earth-Moon system," Celestial Mechanics and Dynamical Astronomy, vol. 108, no. 3, pp. 301-313, 2010.

[10] À. Jorba, "A numerical study on the existence of stable motions near the triangular points of the real Earth-Moon system," Astronomy and Astrophysics, vol. 364, pp. 327-338, 2000 .

[11] J. Simpson, "Dust-cloud moons of the Earth," Physics Today, vol. 20, no. 2, pp. 39-46, 1967.

[12] J. Roach, "Counterglow from the Earth-Moon libration points," Planetary and Space Science, vol. 23, no. 1, pp. 173181, 1975.

[13] M. Winiarski, "Photographic observations of the cloud in the neighbourhood of libration point L5 of the Earth-Moon system," Earth Moon Planets, vol. 47, no. 2, pp. 193-215, 1989.

[14] J. Slíz-Balogh and A. Barta, "Gábo Horváth, "Celestial mechanics and polarization optics of the Kordylewski dust cloud in the Earth-Moon Lagrange point L5 - part II. Imaging polarimetric observation: new evidence for the existence of Kordylewski dust cloud"," Royal Astronomical Society, vol. 482, no. 1, pp. 1$15,2019$.
[15] N. S. Moeed and J. C. Zarmecki, "Feasibility of space based observations of the Kordylewski clouds," Advances in Space Research, vol. 20, no. 8, pp. 1527-1530, 1997.

[16] A. Kliore, "The utility of libration point satellites," Tech. Rep. 33-154, JPL Technical Memorandum, 1963.

[17] J. T. Wheeler, Alternate Apollo missions - libration points, 1967.

[18] K. Uesugi, "Results of the MUSES-A "HITEN" mission," Advances in Space Research, vol. 18, no. 11, pp. 69-72, 1996.

[19] R. Laufer, W. Tost, O. Zeile, R. Srama, and H. P. Roeser, “The Kordylewski clouds- an example for a cruise phase observation during the lunar mission BW1," in 11th ISU Annual International Symposium, Strasbourg, 2007.

[20] E. Glenn Lightsey, "CubeSat Formation Flying," Workshop on CubeSat-based Low Frequency Radio Astronomy Missions, 2012.

[21] X. Hou, J. Tang, and L. Liu, "Several possible mission orbits around Earth-Moon triangular libration points," in 30th ISTS Conference, Kobe-Hyogo, 2015.

[22] M. A. Covington, Astrophotography for the Amateur, Cambridge University Press, Cambridge, UK, 1999, ISBN 0521641330 .

[23] D. C. B. Whittet, Dust in the Galactic Environment, Institute of Physics (IOP) Publications, Bristol, 2nd edition, 2003.

[24] K. Kordylewski, "Photographische untersuchungen des librationspunktes L5 im system Erde-Mond," Acta Astronomica, vol. 11, p. 165, 1961.

[25] $\mathrm{H}$. Wu, Polarization of scattering light in the atmosphere, Master, Hefei University of Technology, 2010. 